\author{
REVISTA ECONOMÍA \\ Vol. $72, \mathrm{~N}^{\circ}$ II6 (noviembre 2020), I03-II8 \\ ISSN-i I390-6380, ISSN-e 2697-3332 | https://doi.org/10.29166/economia.v72i116.2623
}



\title{
FINANZAS SOLIDARIAS Y EL FORTALECIMIENTO DE LOS CIRCUITOS ECONÓMICOS SOLIDARIOS INTERCULTURALES EN LA COMUNIDAD DE OYACACHI, ECUADOR
}

\author{
SOLIDARITY FINANCE AND THE STRENGTHENING OF INTERCULTURAL \\ SOLIDARITY ECONOMIC CIRCUITS IN THE COMMUNITY OF OYACACHI, ECUADOR
}

\author{
ROMÁN DIEGO MEJÍA, WITHNEY OLMEDO, ÉDGAR BENÍTEZ \\ Investigadores independientes
}

\begin{abstract}
Recepción del manuscrito: 29 de septiembre de 2020
Aceptación versión final: 21 de noviembre de 2020
\end{abstract}

\begin{abstract}
RESUMEN El trabajo presenta el caso de finanzas solidarias (Fs) en una de las experiencias sistematizadas dentro del Grupo de Trabajo de Economía Social y Solidaria-UCE, de la comunidad de Oyacachi y de su caja de ahorro (CA), su desarrollo y constitución. Así como su influencia en los distintos avances de la comunidad en donde opera. Para ello, se utiliza el enfoque de análisis de los circuitos económicos solidarios interculturales (CESI) que posibilita exponer las diferentes dimensiones cultural, política, ambiental y económica, del proceso y su base de economía solidaria (Es), soportados en una metodología cualitativa y se enfatiza en la importancia e impacto de las Fs y su práctica, que han posibilitado conectar y fortalecer a esta comunidad.
\end{abstract}

PALABRAS CLAVE Economía solidaria, circuitos económicos solidarios interculturales, finanzas solidarias.

ABSTRACT The paper presents the case of solidarity finance in one of the systematized experiences within the Social and Solidarity Economy Working Group-UCE, that of the Oyacachi community and its savings bank, its development and constitution, as well as its influence on the different advances of the community where it operates. For this, the analysis approach of intercultural solidarity economic circuits (CESI) is used, which makes it possible to expose the different cultural, political, environmental, and economic dimensions of the process and its basis of solidarity economy ( $\mathrm{SE}$ ), supported by a qualitative methodology and emphasizes the importance and impact of Solidarity Finance (FS) and its practice, which have made it possible to connect and strengthen this community.

KEYWORDS Solidarity economy, intercultural solidarity economic circuits, solidarity finance.

JEL CODE A13, B59, R51, B55 


\section{INTRODUCCIÓN}

La economía social y solidaria (Ess) surge como una reinterpretación crítica de los procesos económicos, vincula a la actividad económica con la actividad humana. Es decir, la actividad económica no es una esfera aislada, que se sobrepone sobre lo social, político y lo ecológico, en palabras de Laville (2015), es un sitio de relación e interdependencia entre el hombre y los medios naturales para garantizar la vida. Así, el análisis de experiencias económicas alternativas a la economía de mercado, permite explorar propuestas y nuevas opciones para la construcción de otra economía, la cual parte desde el colectivo y se distancia del individualismo como práctica convencional, estas experiencias de otra economía permiten visualizar hechos sociales, culturales y económicos diferentes a los ya establecidos y controvertirlos al presentar nuevas e importantes experiencias.

Es relevante observar que la comprensión de los aspectos culturales, sociales, geográficos, ecológicos, territoriales, entre otros, son centrales a la hora de proponer alternativas frente a los modelos ortodoxos, ya que la vida humana, desde la Ess, es asumida dentro de un contexto más complejo, articulado y dinámico, no solo dependiente o determinado por las leyes de la oferta y la demanda. En donde es central observar, como lo señala Silva citando a Razeto, lo «asociado a la colaboración, la asociatividad, la participación de la comunidad y la acción solidaridad» (Silva Urbina, 2013, p. 66), lo que se define como el factor c. Estos elementos vinculan principios éticos que buscan otro camino para la vida humana, e implican una asociación y trabajo más allá del que predomina en las lógicas del capital.

Actualmente, la pandemia de covid-19 ha hecho manifiesta la inoperancia del modelo ortodoxo, evidenciando que no hay respuestas claras desde el sistema dominante, ante lo cual la acción social muestra otro camino. Miles de personas han puesto a marchar iniciativas solidarias para hacer frente a los cambios que se han suscitado, ${ }^{1} \mathrm{y}$ la Ess se posiciona como alternativa real de transformación y construcción de nuevas relaciones entre las personas, con el medio ambiente y formas sostenibles y sustentables de desarrollo.

La situación actual muestra que la Ess, dadas sus características expuestas por el Movimiento de Economía Social y Solidaria del Ecuador (MESSE), es una alternativa dinámica y potente que debe ser considerada para salir de la crisis, conectar a amplios sectores sociales para apoyarse, crecer y fomentar alternativas de participación más democráticas que sometan a la economía para cumplir con su finalidad real, que es la de proveer a la sociedad de forma sostenible, los recursos materiales tanto a nivel individual como colectivo, para su desarrollo integral y en equilibrio con el medio ambiente (Urretabizkaia y Fernández-Villa 2015). En este sentido, en este estudio se adhiere a la propuesta colectiva del MESsE, que señala que:

La economía solidaria pretende incorporar a la gestión de la actividad económica los valores universales que deben regir la sociedad y las relaciones entre toda la ciudadanía: equidad, justicia, fraternidad económica, solidaridad social y democracia directa. Y en tanto que una nueva forma de producir, de consumir y de distribuir, se propone como una alternativa viable y sostenible para la satisfacción de las necesidades individuales y globales, y aspira a consolidarse como un instrumento de transformación social. (Urretabizkaia y Fernández-Villa 2015, p. 40) 
En esta perspectiva, se considera que la propuesta conceptual y metodológica de los circuitos económicos solidarios interculturales (CESI) es indispensable para pensar y articular opciones válidas de transformación en tiempos convulsos como los que se viven. De esta manera, los CESI «son espacios de articulación de las prácticas y actores de la Es relacionados a los campos de: producción sana, Fs, el comercio justo, el consumo responsable, los que realizan posconsumo, el turismo comunitario, la salud ancestral» (Jiménez, 2014, p. 2), que además se sustentan en los principios de la ess y parten de organizarse con la finalidad de dar respuesta a sus necesidades más fundamentales y posibilitar formas sostenibles de vida como lo plantea Jiménez (2014).

Los CESI con su análisis dimensional político — relaciones de poder entre los participantes y con el contexto-, económico — relaciones de asociación para producir entre la comunidad y las redes de intercambio-, ambiental — relaciones con la naturaleza - y cultural construcción en el territorio de identidad cultural- (Urretabizkaia y Fernández-Villa 2015), posibilitan conceptuar la realidad social y presentar los impactos del trabajo conjunto de la comunidad y sus logros, como la cotidianidad de la incorporación a los procesos productivos de los principios de complementariedad, redistribución, vincularidad e interculturalidad (Jiménez, 2014, p. 3), como también la equidad de género y generacional y a su vez la construcción de la autonomía en el territorios (Urretabizkaia y Fernández-Villa 2015, pp. 48-49). Finalmente, existe un aporte político clave que para el caso colombiano se lo ha expuesto relacionado con el concepto de redes de colaboración solidaria (RCS), vinculadas estrechamente con los CEsi, así:

Políticamente, estas redes implican la territorialización que, de acuerdo con Matta, Magnano, Orchansky y Etchegorry (2013), se entiende como proceso sistemático que fortalece-empodera a los actores locales, cuando el crecimiento económico se concibe como una espiral ascendente con un fuerte componente de participación ciudadana, caracterizado por poseer coherencia y personalidad propia [...]

El vínculo ético de las RCS reivindica principios, valores y saberes ancestrales vitales para el sostenimiento armónico de las relaciones sociales, económicas, políticas y culturales de los agentes que integran el territorio. (Castañeda et al., 2016, p. 44)

Desde esta perspectiva, se aborda la experiencia localizada en Oyacachi, en donde distintas formas de ess se han consolidado, fomentando procesos de intercambio, producción local agropecuaria, artesanal y de Fs, entre otros, en un territorio particular. En este recorrido, se expondrán los hallazgos que el trabajo de campo ${ }^{2}$ ha evidenciado, el cual tuvo por objetivo visibilizar los vínculos sociales que se han generado en la comunidad de Oyacachi a partir de las experiencias solidarias de la caja de ahorro y crédito desde el enfoque de las finanzas populares y solidarias, y en donde la experiencia de la Asociación de Apoyo Solidario Randipurashun ha permitido potenciar el crecimiento de varias de las alternativas que la comunidad ha construido en su proceso. Para esta finalidad, la sistematización de esta experiencia utilizó como metodología un análisis crítico y descriptivo mediante un estudio de caso, ubicándose en la perspectiva científica interpretativa y complementando el proceso con instrumentos de recolección de información cuantitativa y cualitativa, que implicó la construcción de indicadores acorde a 
la realidad, como la valoración y revisión de bibliografía pertinente. Finalmente, para su aplicación, se recurre a los grupos focales, las encuestas y entrevistas, reflejadas en este trabajo.

Por otra parte, la experiencia de la caja comunal de Oyacachi, se inscribe en una propuesta transformadora de las finanzas tradicionales y se incorpora en la finanzas populares y solidarias (FPS) las cuales, según (Montalvo y Jacome, 2019), se organizan bajo supuestos diferenciados de las finanzas convencionales, su accionar nace del compromiso de sostener, ampliar y legitimar los procesos de participación democrática e igualitaria de los socios y garantizar la sostenibilidad económica de la organización mediante el desarrollo de productos financieros que respondan a las necesidades y requerimientos de la asociación y el entorno cultural y social en el que operan.

La disputa del sentido social de las finanzas, y sobre todo, la relación de éstas con el desarrollo, desde los años 60 han pasado por diversas modalidades que comenzaron con el crédito rural hasta los años 70, pasando al microcrédito y posteriormente desplazándose a la industria de las microfinanzas en los años 80 y 9o, y en el último tiempo desde una perspectiva heterodoxa, al proceso de fomento de las finanzas populares y solidarias como parte de los desarrollos constitucionales de 2008 en el país (Orellana, 2014). Estos cambios, que se contraponen a la concepción ortodoxa de las finanzas, que hizo agua con los procesos de endeudamiento que generaron, sobre todo en épocas neoliberales, y que finalizan con la crisis de 1999 en el país, es donde las cooperativas de ahorro se convirtieron en un actor relevante a nivel financiero ( $\mathrm{Da}$ Ros, 2003), y llevan a replantear la función de las finanzas, su importancia social, su perspectiva ética y su uso solidario para consolidar procesos territoriales como alternativas a la propuesta tradicional, en donde sus actores y lucha han sido fundamentales.

El caso de la comunidad de Oyacachi, en donde su experiencia los llevó a constituir un proceso de Fs, y que ha permitido apalancar otras alternativas económicas apoyadas en principios solidarios, es un ejemplo que aporta a la discusión y abre caminos para construir alternativas.

\section{BREVE RESEÑA HISTÓRICA DE LA COMUNIDAD Y CAJA DE AHORRO}

En el Ecuador, las comunas indígenas se caracterizan por ser, casi en su totalidad, territorios ancestrales y poseer una riqueza cultural que le ha permitido permanecer en el tiempo, «hay alrededor de 1800 comunas en la cierra, de las cuales 1513 tienen reconocimiento jurídico del Estado» (Villavicencio Loor, 2002, p. 5). Así, según Jacome (2019), la conformación de la comuna es una forma de organización predominante llevada a cabo por grupos indígenas en respuesta a los contextos de modernización por parte del Estado a mediados del siglo xx. Las comunas ponen en práctica muchos ideales propios de la comunidad indígena, entre lo más destacado se encuentra la propiedad comunal, donde se incluye la democratización de la tierra y la vivienda, como derechos propios de los comuneros y no mercancías exclusivamente, la autonomía indígena y la democracia participativa (Rayner, 2019).

La comuna de Oyacachi, del kichwa yaku llakta o tierra del agua, está ubicada sesenta kilómetros al nororiente de El Quinche y a unos treinta y cinco kilómetros al sur-oriente de Cangagua, a una altitud de unos 3050 m. s. n. m. Es una comunidad indígena ancestral con alrededor de 150 familias, que posee un registro histórico de su asentamiento a mediados del año 
1559 (Carranza, 1994). Es decir, más de 500 años la comunidad de Oyacachi existe entre las estribaciones orientales de la Cordillera de los Andes en el cantón el Chaco, provincia del Napo. Su conformación como comuna ha transitado por varios hitos históricos, iniciando desde su reconocimiento en (1906), la constitución legal como organización indígena (1939); la constitución jurídica de la comuna (1948); el descubrimiento de las termas (1950); la reubicación de la comuna (1970); la consolidación de la reserva natural Cayambe-Coca (1970); la fundación de iglesias evangélicas (1972) y católicas (1983) y su reconocimiento legal actual como comuna, que es dado mediante el acuerdo número 193 del 21 de junio del 2006 concedido por el Consejo de Desarrollo de las nacionalidades del pueblo del Ecuador (CONDEPE).

Según Rayner (2019), la conformación comunitaria y la instauración de sus instituciones han sido llevados a cabo o dirigidas por instancias internas elegidas por ellos mismos, así el cabildo conformado por individuos involucrados en la defensa, organización y representación de los intereses comunitarios, permiten cultivar relaciones comunales y mantener las tradiciones y compromisos que están en peligro de desaparecer. El cabildo de la comuna de Oyacachi y la Asamblea General son las instancias máximas de representación que poseen las competencias de mediación, resolución, apoyo y ejecución que busca el bienestar colectivo. Además de generar vínculos entre los diferentes miembros y actores de la comunidad, ya que toda decisión es presentada y debatida en la Asamblea General. (Curichumbi, 2012).

Desde hace más de 500 años, la comunidad de Oyacachi ha sido una zona dedicada a la ganadería, el comercio y las artesanías, lo que les ha permitido sostener su economía de subsistencia y autoconsumo, donde participan hombres y mujeres de la comunidad, lo que ha posibilitado crear alternativas de producción, comercialización, distribución y generación de ingresos propios. Según Carranza (1994), las actividades de comercio de la comunidad surgieron, en un primer momento, por ser zona de tránsito entre la Sierra y el Oriente y por su riqueza natural al estar rodeada de bosques y afluentes de ríos; posteriormente por la presencia de la virgen de El Quinche que permitía la afluencia de diversas personas y comunidades aledañas, como de la población de Cayambe.

La experiencia de la comunidad, que lleva a la constitución de la caja de ahorro (CA), es producto de prácticas previas que fracasaron, ya que en un inicio los principios que orientaban su actividad económica se sustentaban en la competencia desleal entre comuneros por tratar de vender sus productos. Así, un ejemplo de ese tiempo fue en el sector de las artesanías, en donde enfocándose en la reducción del precio como estrategia de comercialización y competitividad, les llevó a que la calidad de las mismas decaiga. Para solucionar el conflicto, la asamblea general de la comuna desarrolló estrategias de asociatividad entre ellos, como la fijación de precios y en el manejo del dinero de las actividades económicas, que se canalizó por medio de la CA. Según Janeth Parión cuenta: «los directivos se pusieron de acuerdo, al principio [y] se formó una directiva [...]; y después se creó los códigos personales, el sistema [...] para poner los precios a los productos. Y [...] fue un éxito» (Parión, 2020). Así, la asociatividad ha permitido el establecimiento y fortalecimiento de varias asociaciones como las asociaciones ganadera y piscícola, que registran sus años de surgimiento alrededor del 1997 y reconocidas en el año 2010; el sector artesanal en el año 2005 y el turismo comunitario en 1999, pero constituida legalmente en el 2009. 
Actualmente, la comunidad ha centrado su actividad económica alrededor del turismo de los espacios comunitarios dentro de su localidad, con la participación de la totalidad de las familias, las cuales se organizan en los sectores de las artesanías (24\%), espacios de las termas $(20 \%)$, ruinas arqueológicas $(16 \%)$, restaurantes (14\%), hoteles (10\%) (Encuesta, 2020$).^{3}$ Dichas actividades mantienen vivos los vínculos entre comuneros al organizarse para cuidar y limpiar sus propios espacios comunitarios por medio de la realización de mingas, ${ }^{4}$ en las cuales participa el cien por ciento de los comuneros, en la práctica de prestamanos el $58 \%$ y el randy randy (dando-dando) con el $38 \%$, y un $4 \%$ que no participa ni de las actividades comunitarias, ni económicas, ni comerciales.

Dichas relaciones dentro de la comunidad de Oyacachi son dirigidas y se mantienen gracias a su estructura organizativa, la cual es coordinada y liderada por el cabildo, y sus decisiones discutidas y aprobadas en asamblea general; $y$, a la vez, con el soporte que brinda la CA propia de la comunidad indígena, que permite financiar las actividades económicas de emprendimiento de los comuneros, canalizando el ahorro en crédito dentro de la comunidad.

La CA de Oyacachi denominada Asociación de Apoyo Solidario Randipurashun, que en kichwa significa «dando y dando», inicia sus operaciones en el año 2008 con alrededor de 69 socios y aportaciones individuales de USD 20 mensuales, logrando un capital USD 1320. Esta base permitió la creación de un fondo de apoyo a ciertas actividades localizadas en la asociación de artesanos y al turismo comunitario, después se sumarían otras franjas económicas como la piscícola (Curichumbi, 2012). Así, Diego Parión, por ejemplo, cuenta que:

[L]a ganadería es una de mis primeras actividades hoy, hago quesos, pero estoy también en la venta de truchas. En las termas se puede vender algunas cositas [...], pero es poco, se deben hacer más cosas, como invertir en piscinas de truchas, y la cA da crédito para capital necesario para comprar. (Parión, 2020)

Para el año 2011, siguiendo la Ley Orgánica de Economía Popular y Solidaria, la asociación de apoyo solidario se establece como Caja Solidaria de Ahorro y Crédito Randipurashun, cumpliendo los requerimientos de las instituciones de control como la Superintendencia de Economía Popular y Solidaria (sEPs), afianzando su objetivo en la necesidad de direccionar los recursos financieros para la sostenibilidad de las diferentes actividades económicas desarrolladas por los comuneros y sus organizaciones y gremios productivos. Así, el tejido productivo y social de la comuna fue consolidándose con procesos de apoyo interno y el fortalecimiento de la administración directa de los recursos de las actividades económicas dentro de la localidad. Esto ayudó al mejoramiento de los ingresos de los comuneros, a mejorar la calidad de vida de las familias que fueron saliendo de la pobreza, como también al afianzamiento de las organizaciones y de la cultura financiera, ya que la dinámica de la CA, respaldada bajo la dirección de un consejo administrativo elegido en la asamblea general de la comuna, en el cual participan como integrantes miembros del cabildo, ha dinamizado la recirculación de los recursos financieros para potenciar el impacto social de los beneficios del ahorro.

En la actualidad, según los datos obtenidos en la encuesta realizada en la comunidad y a los socios de la CA, se conoce que ésta tiene un respaldo mayoritario del 9o\% de las familias de la comunidad, las cuales participan como socios activos de la misma, el $64 \%$ de los encuestados 
realizan su ahorro desde la cantidad USD 25 hasta USD 50 en la caja; el acceso al crédito es diversificado para todos los socios, y solo el 18\% apuestan por otros oferentes financieros, como bancos privados y cooperativas de ahorro y crédito, fuera de la comunidad.

Según la información recopilada sobre el uso del crédito, se conoce que el 67\% de los créditos solicitados por las familias a la CA, se destinan a la compra de activos productivos necesarios para sostener y ampliar sus actividades económicas e iniciar nuevos emprendimientos, mientras un $2 \%$ destina su crédito al consumo, un $6 \%$ a salud y un $4 \%$ a educación. Además, el trabajo de campo permite señalar que los comuneros que participan de la CA en un $76 \%$ consideran muy importante el desempeño de la caja por la rapidez al otorgar un crédito, y el $72 \%$ considera muy importante la confianza depositada en ésta para pedir o gestionar un crédito; el 68\% considera importante el menor número de trámites y exigencias que se les solicita a comparación de la banca tradicional.

De esta manera, la ca de la comunidad marca un precedente como alternativa de organización en el trabajo, tanto como en las relaciones económicas y financieras, basadas en principios inscritos en la economía social y solidaria, en las cuales se priorizan la convivencia del ser humano con su entorno ecológico, las relaciones de producción solidaria que permite el desarrollo de la vida, sin pretensiones competitivas, ya que la misma se encuentra subordinada al principio de la solidaridad, provocando relaciones de complementariedad entre los individuos y distanciándose del enfoque de la economía ortodoxa, regida desde las fuerzas de oferta y demanda del mercado.

La conformación de estas otras formas de organización financiera locales, iniciadas por la autogestión, posibilitan la integración social, el fomento de la cultura cooperativista y fortalece los pilares del compromiso, la confianza y la implicación social que permiten a la población participar de su propia historia, de posibilitar la propiedad social y la gestión del control (Orellana, 2014). Así, la comunidad de Oyacachi se propuso la democratización de los recursos financieros bajo iniciativas de la autogestión a través de su cA para la satisfacción de las necesidades propias de la localidad, todo aquello enmarcado en los principios de colectividad y unidad (Curichumbi, 2012), los cuales permiten que los comuneros se integren y mantengan un entorno social activo; así, la reinversión del ahorro en la localidad en créditos productivos y a bajas tasas de intereses, han fortalecido a los emprendimientos y a las asociaciones, creando fuentes de trabajo, cultivando la identidad de los comuneros, poniendo las finanzas al servicios de todos y robusteciendo su territorio.

FORTALECIMIENTO DE LAS DIMENSIONES POLÍTICA, ECONÓMICA, CULTURAL Y AMBIENTAL A TRAVÉS DE LOS CIRCUITOS ECONÓMICOS SOLIDARIOS EN LA COMUNIDAD DE OYACACHI

La parroquia de Oyacachi (Napo), conformada por una comunidad indígena de habla kichwa, se encuentra ubicada dentro del Parque Nacional Cayambe Coca (PNCC) y su economía se fundamenta en la producción agropecuaria y turística principalmente.

Esta comunidad ha sumado a sus prácticas cotidianas la economía social y solidaria como modelo alternativo de desarrollo socioeconómico local. Entendiendo que Ess consiste en actividades económicas que toman en cuenta a las personas, el medio ambiente, el desarrollo 
sostenible y sustentable por encima de otros intereses (Coraggio y Laville, 2016), aplicando estrategias o herramientas de tipo operacional, como son los CESI, los que tienen en cuenta a los actores en el territorio y sus prácticas económicas que articulan la producción sana, las finanzas solidarias, el comercio justo, el consumo responsable, el posconsumo, el turismo comunitario, la salud ancestral, entre otras (Urretabizkaia y Fernández-Villa, 2015, p. 45), formando CEsi con el fin de potencializar el tejido social de una colectividad, favoreciendo de esa manera la construcción del buen vivir en un territorio (Silva Urbina, 2013, p. 53).

La comunidad, identificó la necesidad de «mejorar la capacidad organizativa, institucional local para el buen manejo de los Recursos Naturales de Oyacachi» (E. Parión, 2019), para lo cual constituyeron la CA Randipurashun, la misma que ha reorganizado el manejo de las actividades económicas, intentando superar la dependencia de la economía de mercado con la ampliación de su capacidad de producción, que a su vez, permita satisfacer las necesidades comunitarias, promoviendo un desarrollo endógeno y vinculado a la Ess.

Este desarrollo local parte de la contextualización del territorio específico, en el que no solo se observan las características y procesos que involucra la dimensión económica que plantean los CESI, siendo necesario relacionar y transversalizar con las dimensiones política, cultural y ambiental que son parte del proceso local en su integralidad y crecimiento.

Para comprender cómo la comunidad de Oyacachi ha integrado y aplicado su modelo de desarrollo local, es imprescindible analizar la forma en la que la constitución de la cA favoreció la reorganización de las relaciones y vínculos comunitarios que incidió en la articulación de los CESI, desde donde se proyecta este análisis con la herramienta dimensional — política, económica, cultural, ambiental-.

\section{LA DIMENSIÓN POLÍTICA}

La organización político-administrativo de la comunidad de Oyacachi tiene dos niveles de autoridad, aquella derivada del Estado, como la junta parroquial, la tenencia política, el registro civil y otros estamentos estatales; y las de la organización comunitaria, como la asamblea general, conformada por toda la población, y el cabildo integrado por cinco representantes elegidos por la asamblea, este último es el brazo ejecutor de las decisiones dictadas por la asamblea general.

Se debe señalar, que todos los aspectos relacionados con el desarrollo comunitario, con la mediación y resolución de conflictos son potestad de la asamblea y su cabildo (Curichumbi, 2012, p. 48). Es decir, que la asamblea general es la máxima autoridad político-administrativa en la comunidad.

En el trabajo de campo realizado en la comunidad, mediante encuestas y entrevistas a 50 representantes de familias que constituyen alrededor del 10\% de la población total, se observó que el $88 \%$ de los encuestados afirmó que la toma de decisiones se realiza de manera consensuada e informada. Además, que los niveles de participación llegan al 98\% de manera muy activa y positiva, dejando solo un $2 \%$ de personas que afirman que su participación es limitada. En una entrevista realizada a uno de los miembros de la asamblea, se señala que «si ahorita mismo soy miembro del cabildo, entonces hemos estado siempre trabajando bien, siempre ha estado pendiente [...] y así para poder salir hacia adelante» (Asociado de la Asociación de 
Ganaderos, 2020). Esto demuestra que la comunidad, a través de la asamblea general y su cabildo, mantiene su autonomía respecto de las entidades gubernamentales con una participación considerable de la población y una democracia, directa y activa, para la toma de las decisiones, permitiendo que la fluidez de la acción organizativa y política sea orgánica.

En este sentido, es relevante destacar que una de las decisiones adoptadas por la asamblea general y ejecutada por su cabildo fue la conformación de la CA, que debía regirse y se rige bajo los principios comunitarios de:

Respeto a las autoridades propias o cabildos, equidad de género, solidaridad (ayuda de bonos y créditos), reciprocidad, redistribución, transparencia y control social, interculturalidad, tierra y territorio (conservación), trabajo (tomando en cuenta el trabajo familiar, comunal y colectivo), recursos naturales (aprovechamiento), y cultura (el respeto por la diversidad cultural). (Parión, 2019)

La CA, así regida por estos principios, se ha constituido no solamente en un pilar económico dentro de la comunidad, sino que ha permitido retroalimentar a las instancias político-administrativas formando ciudadanos proactivos además de fortalecer los vínculos de participación, organización, administración y confianza comunitarios. Este ejemplo muestra una lógica distinta de construcción y ejercicio del poder, desde abajo, que fortalece todos los niveles de la organización, su autodeterminación, de relacionamiento de sus miembros interna y externamente, como los principios de autonomía y la construcción práctica de sujetos políticos.

\section{LA DIMENSIÓN ECONÓMICA}

La dimensión económica es donde mayor impacto se observa porque en ésta el modelo de Ess que se planteó la comunidad, como una alternativa de desarrollo local ante el abandono estatal y la excluyente economía de mercado, ha mostrado que la cA ha jugado un papel preponderante influyente en la formación de CESI, redes de intercambio, mejorando la producción, el consumo y el ahorro y, por tanto, las condiciones de vida de la población.

La CA produjo la reorganización de las asociaciones preexistentes en la comunidad como la asociación de ganaderos, artesanos, de piscicultura y el centro de turismo comunitario. Al mejorar su organización y administración, favorecieron y fortalecieron emprendimientos como el turismo ecológico, el invernadero 5 para productos agrícolas y la construcción de infraestructura, demostrando la importancia del factor c del desarrollo comunitario de Oyacachi. Según Jiménez (2016), tomando a Razzeto, el factor c se denomina a la organización como fuerza, que permite el levantamiento de proyectos y emprendimientos, que transforma ésta energía base para producir bienes y servicios de mayor calidad.

A la cA de Oyacachi pertenecen cada una de las asociaciones que mantienen una cuenta de ahorros por sí mismas y, a su vez, cada miembro de las asociaciones con una cuenta de ahorros individual. Es decir, que los recursos generados en la comunidad no van al sistema financiero tradicional, como bancos o cooperativas, sino que se quedan en la CA de la comunidad; esto permite que se otorguen créditos productivos, estudiantiles, de emergencia y de vivienda, con un interés entre el $2 \%$ y $7 \%$ anual, mientras que en el sistema financiero los intereses rondan el 16\% y 17\%, incluso más altos. En el trabajo de campo en la comunidad, se pudo determinar 
que la percepción de la gente con respecto a las actividades de la CA y su utilidad es: ayudar a los emprendimientos productivos, generar empleo, fortalecer la asociatividad, apoyar en la infraestructura, fortalecer la confianza entre los socios, fomentar el desarrollo, y también se preocupa por temas de vivienda, salud y educación, obteniendo una respuesta positiva en un $98 \%$ de los encuestados.

En el contexto así descrito, es necesario ejemplificar cómo funcionan los CEs, las Fs, las RCS, la generación de empleo y el consumo; en este sentido, por ejemplo, la asociación de ganaderos produce leche, queso y carne, los mismos que son recolectados por la asociación y comercializados tanto en la comunidad como en mercados externos, como el de Cayambe; del ingreso económico generado por esta venta, cada socio realiza un aporte a la cuenta de la asociación en la CA, el mismo que servirá para cumplir con los principios que rigen su propósito, este tipo de RCs se replica en las asociaciones de piscicultura y artesanías.

Un caso especial es el del turismo, que maneja las termas, el turismo de aventura, arqueológico y gastronómico, ya que el ingreso generado por esta actividad turística es administrado en la CA, y sirve para otorgar créditos y, en parte, para el pago de los salarios de los empleos generados como guías turísticas, personal de mantenimiento, personal administrativo y también el mejoramiento de infraestructura. Otras estrategias que utiliza la comunidad de Oyacachi dentro de su objetivo de desarrollo comunitario local, es privilegiar la participación de jóvenes y niños en las actividades productivas, así como la educación formal, que permitirá el traspaso intergeneracional de las responsabilidades y metas que persigue la comunidad (sumak kawsay).

En el trabajo de campo, se observó que la educación es un punto importante para el crecimiento comunitario, ya que el $46 \%$ de los encuestados tiene una educación de bachillerato, el $4 \%$ educación básica completa y tan solo el $2 \%$ una educación básica incompleta; seguido de una primaria completa con $10 \%$ y de primaria incompleta del $18 \%$, mientras que un $4 \%$ de personas no tienen educación formal, pero saben leer y escribir; y tan solo un $4 \%$ de las personas no reporta tener educación. Con respecto a la educación universitaria, el 12\% de los encuestados tiene este nivel de formación, el expresidente de la junta parroquial, Micael Parión, señala que la generación de talento humano capacitado es muy importante debido a que permite una mejor organización y administración de las actividades económicas, ésta es la razón por la que se apoya a los universitarios que han regresado a la comunidad para que ocupen cargos en las distintas asociaciones y organizaciones según su capacidad y su estudio (Parión, 2020).

Se debe señalar que de la producción total de Oyacachi, según las encuestas realizadas, en un $20 \%$ sirve para el consumo interno y el $80 \%$ es comercializado fuera de la comunidad, en este aspecto, otro de sus objetivos es disminuir la dependencia de mercados externos, privilegiando el consumo local. Sin embargo, el autoabastecimiento de productos e insumos es bajo, por lo que siguen recurriendo a los mercados de Cayambe y Cangagua.

Finalmente, se resalta cómo la ess mediante las Fs de la comunidad, han logrado otorgar un bono económico a las personas jubiladas de la asociación de artesanos de Oyacachi (Parión, 2020), cumpliendo así con otro de sus principios. La CA, así concebida como un ente financiero local y solidario, tiene una serie de limitaciones dentro de las que se puede señalar un presupuesto limitado y totalmente dependiente de la producción de las asociaciones; esto no 
le permite satisfacer en un mayor porcentaje las necesidades y aspiraciones de la comunidad de Oyacachi, ${ }^{6}$ quienes aspiran a créditos más altos y mayores tiempos de plazo para los pagos.

Es por esto que la comunidad ve necesario el fortalecimiento y crecimiento de la CA, para así aportar en el crecimiento de las demás actividades económicas y viceversa, construyendo un tejido social con objetivos unificados que permitan conseguir el buen vivir y a su vez una mejor calidad de vida.

\section{DIMENSIÓN CULTURAL}

Siendo Oyacachi una comunidad kiwcha, relativamente aislada, han mantenido mucho de su cultura ancestral que se demuestra a través de sus costumbres, lenguaje, principios y tradiciones. Dentro de las más relevantes se encuentra la trasmisión oral de sus costumbres, trueque, minga, prestamanos (randy-randy) y salud ancestral; estas costumbres han permitido y facilitado la implementación del modelo económico de desarrollo local solidario, pero a su vez, el modelo de ess implementado en Oyacachi mediante CESI, RCS y Fs ha fortalecido la identidad comunitaria, mejorado la autoestima y logrado una visión colectiva de desarrollo. Se puede observar una imbricación entre los elementos tradicionales de la cultura andina y aquellos solidarios que implica la Ess.

Edison, un comunero entrevistado, muestra que la identidad en el caso del turismo, no solo se encuentra a nivel comunal, sino también personal, dice:

[...] económicamente también se ha mejorado, también se ha trabajado con el turismo, uno empieza a valorar lo que es la comunidad. Por ejemplo, yo soy indígena de habla kiwcha, eso se empieza a valorar más cuando se trabaja en el turismo. Salgo afuera y no me da vergüenza decir que soy indígena de habla kiwcha de Oyacachi. Con orgullo me presento. (Tandayami, 2020)

Hay algunos ejemplos importantes de la valoración y apropiación de su cultura que han permitido mejorar la autoestima y fortalecer la identidad de la comunidad, éstos están en relación con la conservación del patrimonio cultural tangible e intangible y el conocimiento ancestral, como son las Ruinas de Maucallacta, 7 que se ha considerado como patrimonio arqueológico nacional por el Ministerio Coordinador de Patrimonio, la Cueva o Gruta de la Virgen de El Quinche, icono religioso del Ecuador, es parte importante de la historia y asentamiento de la comunidad (ServiCoca, 2015, p. 56). Por último, esto muestra que la propia comunidad tiene claro su proceso y aquello que representan su cultura y organización, que se despliega en un territorio ancestral y un modo de vida particular.

En el trabajo de campo, el 97\% de nuestros entrevistados y encuestados, afirman que su identidad cultural se mantiene con la transmisión oral de la elaboración de artesanías, el cuidado de los animales, la siembra y cosecha de productos, así como el respeto de las autoridades a las decisiones de la asamblea y de la comunidad. Si bien es cierto, es la asamblea la que dicta las políticas que guían a la comunidad en Oyacachi, la CA ha desempeñado un papel fundamental, ya que siendo una organización de carácter económico y administrativo, influye directamente en los principios que rigen la vida comunitaria, la solidaridad, la confianza, la igualdad de género y el compromiso, entre otros, añadiendo algo que es importante en las condiciones actuales, inculcando la cultura de ahorro tanto en los niños como en los adultos. 
En las entrevistas se muestra cuán importante se ha vuelto la cultura del ahorro, por ejemplo, Janeth menciona que «para que ellos también aprendan a ahorrar su dinero [...] mi hijo debe ahorrar para el estudio o de pronto alguna emergencia, de pronto no se sabe» (Parión, 2020). Es así que en las encuestas realizadas se puede observar de manera general que el $92 \%$ de los encuestados consideran importante ahorrar, y que ahorran especialmente para emergencias y necesidades con un $43 \%$, seguido de un $28 \%$ para emprendimientos, un $17 \%$ para salud, un $10 \%$ para consumos y gastos y, por último, un $2 \%$ para capacitaciones; esto demuestra una nueva influencia cultural en donde los elementos ancestrales se enriquecen con los aportes de la cultura de ahorro y solidaria.

\section{DIMENSIÓN AMBIENTAL}

La comunidad de Oyacachi se encuentra ubica en las estribaciones orientales de la cordillera central de los Andes, dentro del Parque Nacional Cayambe-Coca (PNCC), ${ }^{8}$ que es un área protegida con ecosistemas sensibles que van desde el bosque nublado andino a extensos pajonales, con abundantes fuentes de agua y una importante biodiversidad en flora y fauna. Como se sabe, en estas áreas protegidas está prohibida o limitada la actividad humana y el impacto que ésta genera, como la tala de árboles, la caza de animales, la contaminación del agua y también las actividades agropecuarias. Esto ha llevado a la comunidad a negociar las formas de participación dentro de la política pública estatal y, por ende, a ser partícipe y beneficiaria del cuidado ambiental así como a la búsqueda de nuevos emprendimientos productivos que guarden armonía entre la producción y el medioambiente, ya que conocen su territorio.

Cabe mencionar uno de los programas relevantes como es el caso de Sociobosque, que consiste en que la comunidad cuide y proteja el bosque a cambio de recibir recursos económicos por parte del Estado (Parión, 2020). Según el estudio de campo, se determinó que el 90\% de las personas encuestadas cree que éste programa es útil y ha favorecido la conservación del medio ambiente. Dentro de la búsqueda de actividades económicas que generen un menor impacto ambiental y que conduzcan al desarrollo socioeconómico local, se promueve el turismo arqueológico, de aventura, de salud — con las aguas medicinales—, que deben ser ecológicos. En las entrevistas realizadas, se menciona que:

Antes nos dedicábamos a la ganadería, teníamos todos ganado en el páramo, un poco acá y allá, pero ahora nos concentrarnos en el turismo, o sea, de hacer algo de nuevo, porque en la parte del páramo es donde nacen las vertientes, los ríos, inclusive las aguas que van a Quito, por eso debimos quitar los ganados de ahí, poco a poco todos se va involucrando en el turismo. (Tandayami, 2020)

Todos los recursos generados por esas actividades son administrados por la cA, que no solo sirve para créditos a la comunidad, sino que se invierten en el mejoramiento de la infraestructura y de los paquetes turísticos que se ofrecen, además de la generación de nuevos empleos para la gente de la comunidad. Actualmente, como una de las aspiraciones que tiene la comunidad se señala el promover el turismo vivencial, que no solo sería una fuente de ingreso más, sino que promocionaría y daría a conocer la herencia cultural de Oyacachi (Tandayami, 2020). 
La comunidad, al conocer su territorio y los elementos culturales que no pueden comprenderse aparte del ecosistema, plantea alternativas y retos que han sido sorteados por la comunidad, de buena manera hasta el momento y que, por otra parte, siguen siendo asumidos como posibilidad de construcción de nuevas alternativas económicas solidarias para permanecer en su territorio.

\section{CONCLUSIÓN}

Los resultados obtenidos de las entrevistas y encuestas realizados a 50 representantes de familias han demostrado la importancia de las finanzas populares, su poder generador de articulación de nuevos circuitos solidarios y fomento al desarrollo comunitario, tornándose en instrumento de soporte del proceso económico; es relevante también la unidad entre la investigación y los procesos comunitarios, fundamental para abrir nuevas alternativas para pensar la economía y la realidad social y aportarse una a la otra.

En el contexto político-administrativo, económico, ambiental de esta comunidad se destacan los procesos sociales previos, que dan origen a la CA comunitaria, con los conflictos desatados por las dinámicas competitivas y de mercado, que solo fueron subsanados mediante la incorporación y articulación de principios solidarios y económicos alternativos a la vida de la comunidad. El resultado de estos esfuerzos es la caja, y su contundencia social y relevancia queda clara, en donde la administración comunitaria ha permitido acabar con prácticas deshonestas e imponer una ética que fortaleció esta experiencia.

Muchas de sus actividades productivas, de comercialización y consumo, están soportadas por la CA que a su vez, es dirigida por la asamblea general, esto ha permitido que la economía social y solidaria a través de los CESI y las Fs, se hayan articulado con las prácticas ancestrales que mantiene la comunidad, siendo esta forma una alternativa de desarrollo comunitario que ha servido para reafirmar su identidad cultural y autoestima, mejorar su calidad de vida, generando un tejido social fuerte y transformado a muchos comuneros en sujetos políticos.

En cuanto a las debilidades del proceso y sus limitaciones, no se puede perder de vista que la comunidad de Oyacachi, por su ubicación — parque nacional—, aunque contrario a los principios solidarios, considera la ampliación de la frontera agropecuaria para aumentar su producción, que es restringida. También hay dependencia de los mercados de Cangagua y Cayambe y la necesidad de conexión, lo que implica, a futuro, pensar en alternativas para una buena complementación, dado que afectan su autonomía y su autoabastecimiento.

Se requiere observar las posibilidades de conexión con modelos similares dentro de la lógica que plantea la ess y los CESI, sobre todo en cuanto intercambios en capacitación, así como de la innovación e implementación tecnológica en el afianzamiento de proyectos sustentables como el turismo en sus diversas modalidades, esto permitiría atraer inversiones o alianzas con otras organizaciones hermanas, como también estatal, mejorando los ingresos de la CA, que a su vez redirigiría su inversión a otros proyectos, correspondientemente, dando como consecuencia final, el mejoramiento de calidad de vida, la dignidad y una visión de futuro hacia el sumak kawsay para la comunidad. 


\section{NOTAS}

1 En el caso ecuatoriano, para más información, véase https://tiendapichincha.com/; y la segunda, http://www.pichinchacomunicaciones.com.ec/el-programa-canastas-solidarias-de-la-prefectura-depichincha-suma-colaboradores/

2 El trabajo de campo comenzó en el mes de diciembre de 2019 con la realización de entrevistas y visitas exploratorias a la comunidad, para posteriormente en el mes de febrero del 2020 realizar encuestas a 50 representantes de familias de Oyacachi y entrevistas a profundidad a actores clave como la familia $\mathrm{Pa}$ rión, Aigaje, Aguagallo, los cuales proporcionaron información extra de la capturada en la encuesta, que se presenta a lo largo de este artículo. También se realizaron grupos focales a mujeres y hombres para indagar sobre las actividades económicas. El 21 de febrero del 2020 se levantó información a nivel de jefes de hogar, sustentado en el Censo de Población de Vivienda CPV (2010), para conocer la población total y el número de hogares que se aproximaba a los 600 individuos y 123 hogares respectivamente; y se aplicó la técnica de muestreo probabilístico estratificado, delimitando la muestra proporcional de 50 individuos jefes de hogar. El instrumento levantó información socioeconómica de los jefes de hogar, la estructura del hogar, vivienda, actividades domésticas y productivas, fuentes de alimentación, ingreso y producción local, participación de la CA, aspectos culturales, políticos y de identidad de la comunidad. 3 La encuesta forma parte de la sistematización de la experiencia de la comunidad de Oyacachi y se publicará próximamente en el informe final del grupo de investigación de Ess de la UCE.

4 Según (Ferraro, 2004) el sistema de prestamanos, normalmente concierne tareas agrícolas, generalmente la preparación del terreno para la siembra o - más a menudo - la cosecha, pero también otras actividades tales como construir una casa. Al igual que el randy-randy (dando-dando), que puede entenderse desde la reciprocidad, la hospitalidad y la generosidad para sus prójimos y el trueque (intercambio no monetario); éstas son actividades que se realizan entre comuneros, donde se intercambia productos, trabajo o servicios, que apoyan a sus actividades económicas, especialmente en la agricultura (Parión, 2020). La minga es el trabajo comunitario que se realiza para la construcción de infraestructura y la limpieza de la comunidad, especialmente relacionado al turismo y se da dos veces cada mes o cuando sea requerido (Parión, 2020). Estas actividades son parte de una visión general del cuidado y mejoramiento de la comunidad, integrándolos en la preservación de su cultura e identidad.

5 Un caso importante es el de la asociación ganadera y la creación de su invernadero, que fue realizado con fondos de la comunidad y de la asociación, conjuntamente con el apoyo del municipio de El Chaco, que formaron hace dos años esta iniciativa, donde la asamblea ha decidido cultivar frutillas durante esta temporada para la venta fuera y dentro de la comunidad, en la que las ganancias y deudas van para la asociación de ganaderos (Asociado de Asociación de Ganaderos, 2020); este proyecto ha generado nuevos empleos para las personas que atienden y mantienen el invernadero y, al mismo tiempo, ha relacionado a la asociación de artesanos con un emprendimiento totalmente nuevo que es el invernadero, consolidando redes de apoyo solidario entre actividades económicas.

6 Un caso particular e importante que señalar es la posición de las mujeres como socias de la CA y parte de la comunidad, debido a que sus actividades productivas, conjuntamente con su trabajo no remunerado, no permite tener el mismo nivel de inclusión financiera por su condición, como es el caso de Janeth, que es cabeza de hogar, madre soltera y artesana y, sin embargo, por esta posición social no puede acceder a créditos más altos que le permitan hacer emprendimientos y mejorar su calidad de vida (Parión, 2020).

7 «Es el pueblo antiguo donde vivía la población de Oyacachi, cuentan sus moradores que se trasladaron a vivir más hacia la parte norte, porque muy cerca de allí se encontraba una grieta que podía derrumbarse y sepultar a todo el pueblo» (Ruinas de Maucallacta, 2020).

8 El Parque Nacional Cayambe-Coca declarado patrimonio por el Ministerio del Ambiente, para la protección de flora y fauna de Imbabura, Napo, Pichincha y Sucumbíos (Parque Nacional CayambeCoca, Sistema Nacional de Áreas Protegidas del Ecuador, 2020). 


\section{REFERENCIAS}

Asociado de la Asociación de Ganaderos. (2020). [En persona]. Oyacachi-Ecuador.

Carranza, C. (1994). Geografía sagrada, espacialidad y organización del cosmos: el caso de CangaguaOyacachi-Quinche. Departamento de Antropología Facultad de Ciencias Humanas, PUCE.

Coraggio J. L. y Laville J. (2016). Economía social y solidaria: conceptos, prácticas y políticas públicas. Hegoa.

Curichumbi, R. (2012). Sistema financiero y solidario de las nacionalidades y pueblos indígenas del Ecuador. El caso de la comuna de Oyacachi-pueblo kayambi. (Tesis de maestría). Quito-Ecuador: Universidad Central del Ecuador.

Da Ros, G. (2003). Cooperativas de las Américas-crédito rural y las cooperativas financieras de Ecuador 2001-2020. Cooperativas de las Américas. https://www.aciamericas.coop/ Credito-rural-y-las-cooperativas

Jacome, V. (2019). El proceso fallido de disolución de las comunas urbanas en Ecuador: el caso de Santa Clara de San Millán, 1973-1986. En J. Rayner y J. M. Conde, Las comunas del Ecuador: autonomía, territorio y la construcción del Estado plurinacional (pp. 107-137). IAEN.

Jiménez, J. (2014). Movimiento de economía social y solidaria del Ecuador: circuitos económicos solidarios interculturales. (s. d.).

Laville, J.-L. (2015). Asociarse para el bien común: tercer sector, economía social y economia solidaria. En J.-L. Laville, La economía solidaria (pp. 147-176). Icaria, s. A.

Montalvo, Y. A. y Jácome, V. J. (2019). Elementos para la construccion de un modelo de gestion dirigido a la banca comunal en Ecuador. Revista CIFE, 79(102). https://doi.org/10.15332/22484914.4857

Orellana, E. (2014). Los aspectos financieros del sistema de economía social y solidaria y sector financiero popular y solidario: microfinanzas, finanzas populares y finanzas solidarias. En Diálogos sobre Economía Social y Solidaria en Ecuador Encuentros y desencuentros con las propuestas para otra economía (pp. 283-332). UPV/EHU.

Parión, E. (2020). Entrevista al presidente de la caja de ahorros. [En persona]. Oyacachi- Ecuador.

Parión, E. (julio, 2019). Caja de Ahorros Randipurashu-Oyacachi. [Diapositivas de PowerPoint]. IAEN.

Parión, G. (21 de febrero de 2020). Encuesta recopilacion de informacion sobre las fuentes de ingresos mas importantes de la familia. (E. A. Herrera, entrevistador).

Parión, J. (21 de febrero de 2020). Encuesta recopilacion de informacion sobre las fuentes de ingresos mas importantes de la familia. (E. A. Herrera, entrevistador)

Parión, J. (2020). Entrevista Asociado de la Asociación de Artesanos. [En persona]. Oyacachi-Ecuador.

Parión, M. (2020). Expresidente de la Junta Parroquial. [En persona]. Oyacachi- Ecuador.

Parque Nacional Cayambe-Coca, Sistema Nacional de Áreas Protegidas del Ecuador. (2020). Áreas protegidas. http://areasprotegidas.ambiente.gob.ec/es/areas-protegidas/parque-nacional-cayambe-coca

Rayner, J. (2019). La (re)construcción de la comuna en el Estado plurinacional. En J. Rayner y J. M. Conde, Las comunas del Ecuador: autonomía, territorio y la construcción del Estado plurinacional (pp. 37-69). IAEN.

Ruinas de Maucallacta. (2020). Visita Ecuador. https://visitaecuador.com/ve/mostrarRegistro. php?idRegistro $=27250$

Rúa Castañeda, S., Monroy Flores, V. E., Peñuela Lizcano, J. D., Pérez Villa, P. E., Calderón Ibáñez, A. de C., Arenas Sepúlveda, C. C. y Jiménez Patiño, H. D. (2016). Integración económica solidaria en 
territorio. Aportes a la construcción de modelos y metodologías. Unidad Administrativa Especial de Organizaciones Solidarias, Universidad Cooperativa de Colombia.

ServiCoca. (2015). Actualización del Plan de Desarrollo y Ordenamiento Territorial. Oyacachi.

Silva Urbina, G. (2013). Circuitos económicos solidarios y puesta en valor del patrimonio. (s. d.).

Tundayami, E. (2020). Entrevista Asociado de la Asociación del Centro Turístico. [En persona]. OyacachiEcuador .

Urretabizkaia, L. y Fernández-Villa, M. (2015). Circuitos económicos solidarios interculturales y su contribución a los derechos económicos de las mujeres: el caso de Loja. Upv Hegoa.

Villavicencio Loor, G. (2002). Pluriculturalidad e interculturalidad en el Ecuador: el reconocimiento constitucional de la justicia indígena. (s. d.). 